\title{
EXTREMELY LOW FREQUENCY SIGNAL ACQUISITION, RECORDING AND ANALYSIS
}

\author{
Franz P. Zantis ${ }^{*}$ _ Ján Hribik ${ }^{* *}$ — Daniela Ďuračková ${ }^{* *}$
}

\begin{abstract}
Our environment is permeated by electrical and magnetic alternating waves in the frequency range above the AC voltage of $50 \mathrm{~Hz}$ and also in the radio frequency range. Much attention from the public is given to these waves. Through numerous studies and publications about this type of oscillations and waves it is largely known from which sources they occur and which impact they have. However, very little information could be found about electrical and magnetic alternating waves in the frequency range below $50 \mathrm{~Hz}$. The aim of this research is to demonstrate that these signals exist and also to show how the signals look like and where and when they occur. This article gives an overview of the occurrence of these ELF (Extremely Low Frequencies) signals, their specific properties in view of the time domain and in view of the frequency domain and of the possible sources of these waves. Precise knowledge of the structures of the ELF signals allows conclusions about their potential to cause electromagnetic interference in electronic systems. Also other effects in our environment, eg on flora and fauna could be explained.
\end{abstract}

K e y w or d s: extremely low frequencies (ELF), ELF signals, receiver, signal recording

\section{INTRODUCTION}

Electrical equipment and facilities in Europe operate mainly with $50 \mathrm{~Hz}$ alternating current. It is a known fact that this causes electric and magnetic fields with operating frequency and their higher harmonics in our environment. The environment is thus permeated by electromagnetic waves. In the frequency range above the $\mathrm{AC}$ voltage of $50 \mathrm{~Hz}$, especially in the radio frequency range, much attention from the public is given to these waves. Through numerous studies and publications it is largely known from which sources they stem and which impact they have. The topic of this work are magnetic and electrical oscillations and waves in a lower frequency range, specifically in the range $0 \mathrm{~Hz}<f<25 \mathrm{~Hz}$. There is only little and insufficient information about their presence and occurrence. Extremely low frequency signals will be referred to as ELF-signals in the sequel. In the published papers [1-5] the frequency range is usually extended to higher frequencies. There are many kinds of signals in this extremely low frequency range. Some of them seem to have a natural origin while others resemble communication signals. Independently of their properties some of these signals can be measured in a wide area $(30 \mathrm{~km}$ circle), others just some meters around a certain location. Also, the temporal appearance is very different. Some signals could be detected over years and then suddenly disappeare for some time. Others were only measured on one day for a few hours and then never again.

For analysis of the recorded signals, some kind of frequency transformations are used allowing better to evaluate them even visually by checking the obtained spectra.
Additionally, the signals could be transformed into an audible frequency range. The human hearing is a very good analyzer [6,7]. It can detect signals which are otherwise masked in their spectra. Best results are obtained if both the visual analyzing of the spectra and hearing of the transformed signals are used at the same time.

Figure 1 shows an example of ELF-signals transformed into the frequency range for consecutive instants of time. It is a section of about 20 min duration with the time along the horizontal and the frequency spectra along the vertical axis where the amplitudes are represented by different colours. Dark colour (black $\rightarrow$ blue $\rightarrow$ violet) are low, brighter (red $\rightarrow$ orange $\rightarrow$ yellow) are higher amplitudes. The strong yellow line in the middle is the $16.67 \mathrm{~Hz}$-line of the railway. Depending on the noise that could be heard after the signals were transformed into the audible area, they were given appropriate names.

\section{THE EQUIPMENT}

To minimize the costs, simple self-made air coils [8] were used as sensors, and PC for recording and evaluation of the signals. The amplitudes of the ELF-signals are usually very low while commercial analogue-to-digitalconverter (ADC) needs input values typically between $500 \mathrm{mV}$ and $2 \mathrm{~V}$. Before the digitalisation, the amplification and low pass filtering is therefore necessary. The dynamic range $A$ of a 16 -Bit-ADC is approximately $98 \mathrm{~dB}$, thus the ELF signal must be pre-processed to be able to use most bits of the analogue-to-digital-converter.

\footnotetext{
${ }^{*}$ RWTH Aachen, Physical Institut IIIa, Im Blumenfeld 5, 52477 Alsdorf, Germany, zantis@physik.rwth-aachen.de ${ }^{* *}$ Slovak University of Technology, Institute of Electronics and Photonics, Ilkovičova 3, 81219 Bratislava, Slovakia, hribik@kre.elf.stuba.sk, daniela.durackova@stuba.sk
} 


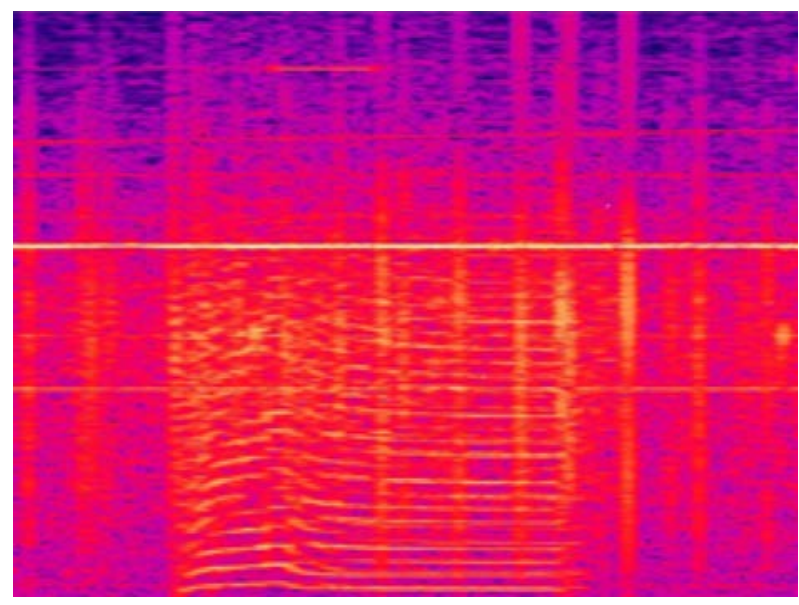

Fig. 1. Example of the ELF signal "Cow": with time long the horizontal, frequency along the vertical axes and colour distinguished amplitude

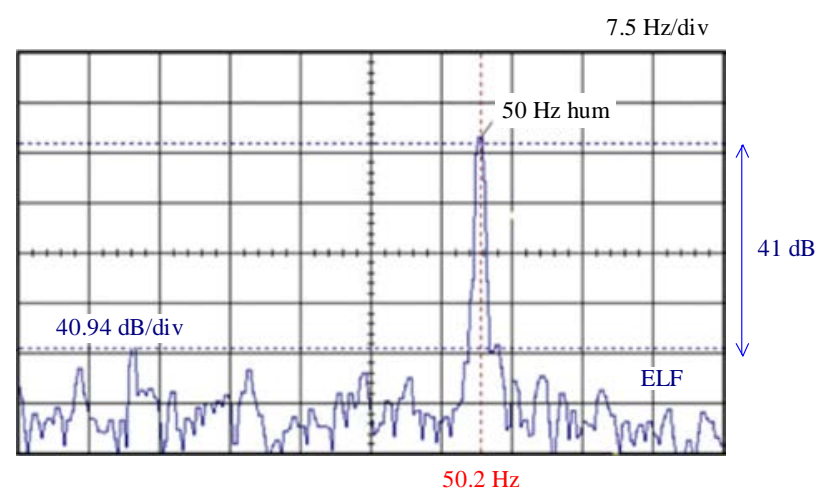

Fig. 2. Spectra of a time-window recorded signal from the sensor (air coil)

The output signal of the used coils contains mainly the $50 \mathrm{~Hz}$ signal of the power supplier and the ELF signals have lower amplitudes. The dynamic range of the receiver system can be increased if a low-pass filter is used which cuts the $50 \mathrm{~Hz}$ signal before the digitization. Figure 2 shows spectra of a time-window measurement. If the $50 \mathrm{~Hz}$ signal is eliminated, the $41 \mathrm{~dB}$ dynamic range is available for the ELF signal.

A secondary effect of filtering the input signal is that a further filter is not necessary to fulfil the Shannon criteria. Additionally, the sample frequency of the ADC can be very low (several hundred $\mathrm{Hz}$, to be on the safe side), which reduces the requirements on the experimental equipment.

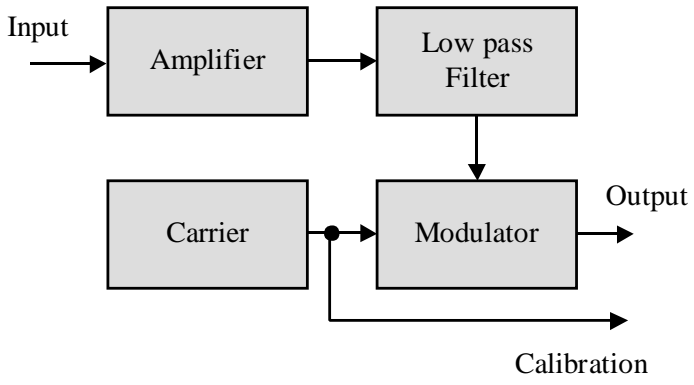

Fig. 3. Recording equipment via the sound card of the PC

After filtering and amplification, two paths were followed. The first equipment uses the sound card of the PC as a frontend (used in the years 2001-2011). The second device uses the USB interface (used since 2011). A schematic overview of the equipment is shown in Figs. 3 and 4. Each PC has a sound card with a 16-Bit-ADC. For that, the signal was modulated on a carrier $(200 \mathrm{~Hz})$. Although the transmission behaviour of the sound cards was not known in details, around of $200 \mathrm{~Hz}$, linearity could be assumed.

Later, the analogue-digital-conversion was made outside the computer and the digital data were transferred via the USB interface. In both cases, the signal ran at first through an amplifier with a linearizer for the sensorcoils and then through a Butterworth low-pass-filter of the 5 -th order.

Since 2010 additional measurements with earth stakes instead of air coil have been made, Fig. 5. When working with earth stakes, complete galvanic isolation between the receiver and the main supply is required. This is best achieved by operating the receiver as well the PC (notebook) on battery supply. In addition, the earth stakes were connected via a signal transformer to the receiver. The used transformer (fy Jensen) is capable of passing very low frequencies.

The whole technical equipment (except the computers) was self-developed and made.

In the first experiments, recording via the sound card and the modulation, Fig. 3, allowed the processing independently of the (not known) properties of the sound card. The software had to provide the recording and demodulation. Since the new hardware using USB is available, just recording of the signals is the software task and due to high computing power of PCs it is possible to calculate also the frequency transform at the same time.

Because of their low frequency the ELF signals cannot be heard directly. Secondly, the recordings are very long

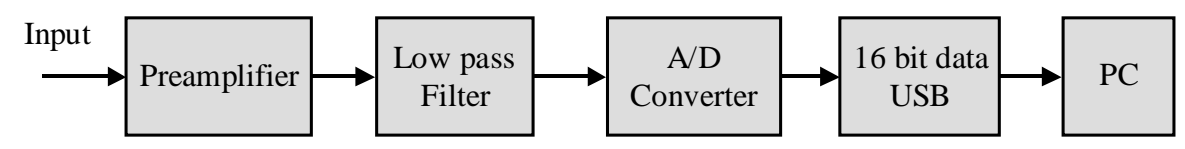

Fig. 4. Recording equipment via USB 


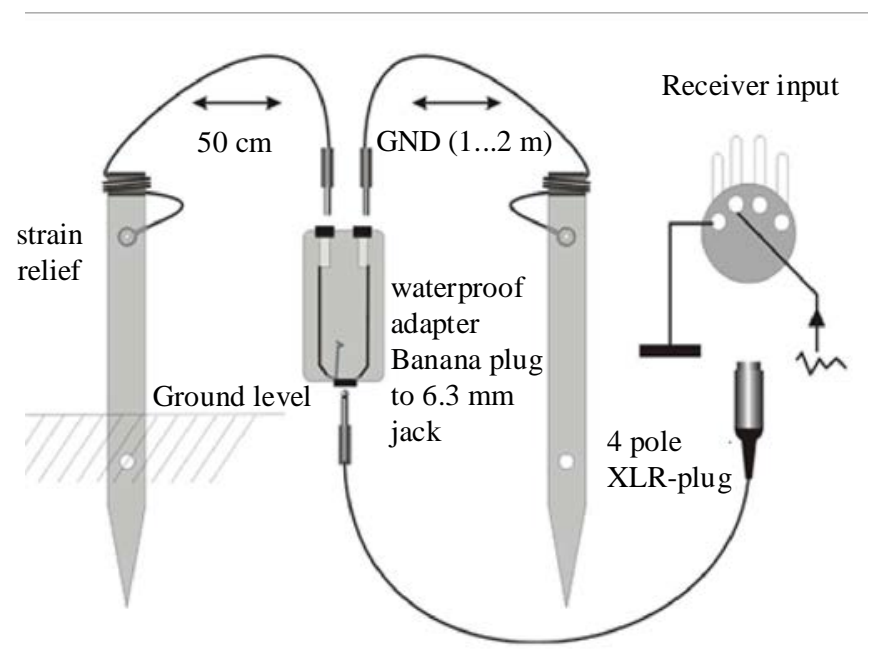

Fig. 5. Self-made ground spikes to use as ELF-sensors

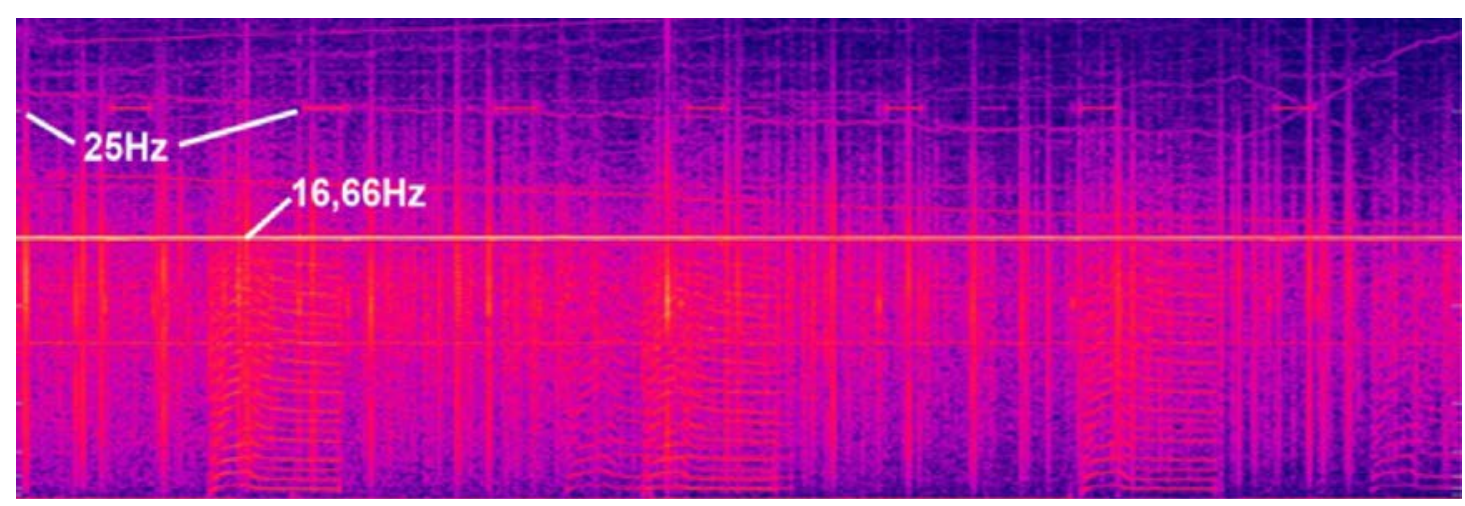

Fig. 6. "Cow" signals recorded during 49 minutes at the north of the mountains "Eifel", frequency vs time; colour — as mplitude

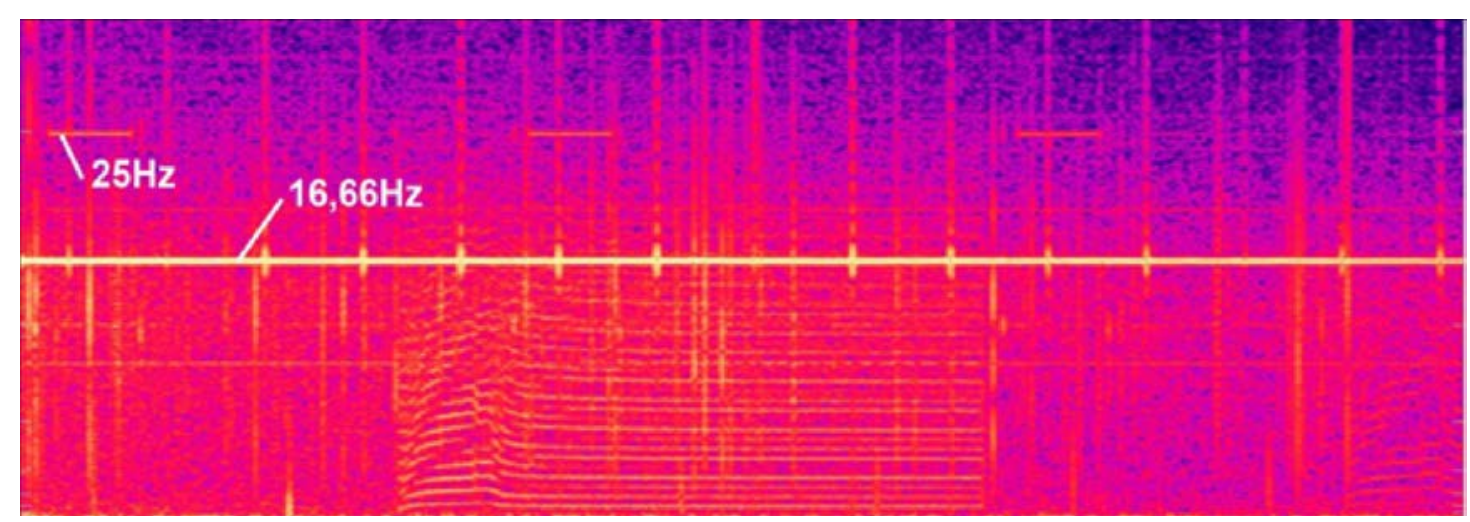

Fig. 7. A single "Cow" signal recorded during 20 minutes at the north of the mountains "Eifel", frequency vs time; colour — as amplitude

(typically from 30 minutes to several hours). To provide the "human ear" analysis the signal must be shifted to a higher frequency range and compressed in time. In a particular case, the files were recorded and saved with a sample frequency $f_{\mathrm{S}}=200 \mathrm{~Hz}$. For analyzing purposes it was transformed to sample rate $f_{\mathrm{T}}=32 \mathrm{kHz}$, whereby a $25 \mathrm{~Hz}$ signal will be heard as $4 \mathrm{kHz}$, and file with an original length of 8 hours ( $e g$ recording over the whole night) can be listened and analyzed in 3 minutes (the shift factor $\left.s=f_{\mathrm{T}} / f_{\mathrm{S}}=160\right)$. For analyzing, at first the freeware
"CoolEdit" was used. Later, since 2013 a self-made analyzer has been used written in Visual Basic.NET.

\section{RECORDING OF ELF SIGNALS}

With the described equipment, lots of recordings were made, beginning in the year 2001. Measurement was made especially at places between Aachen, Cologne, Dremmen and Hürthgenwald in Germany. Further places are Büsum 


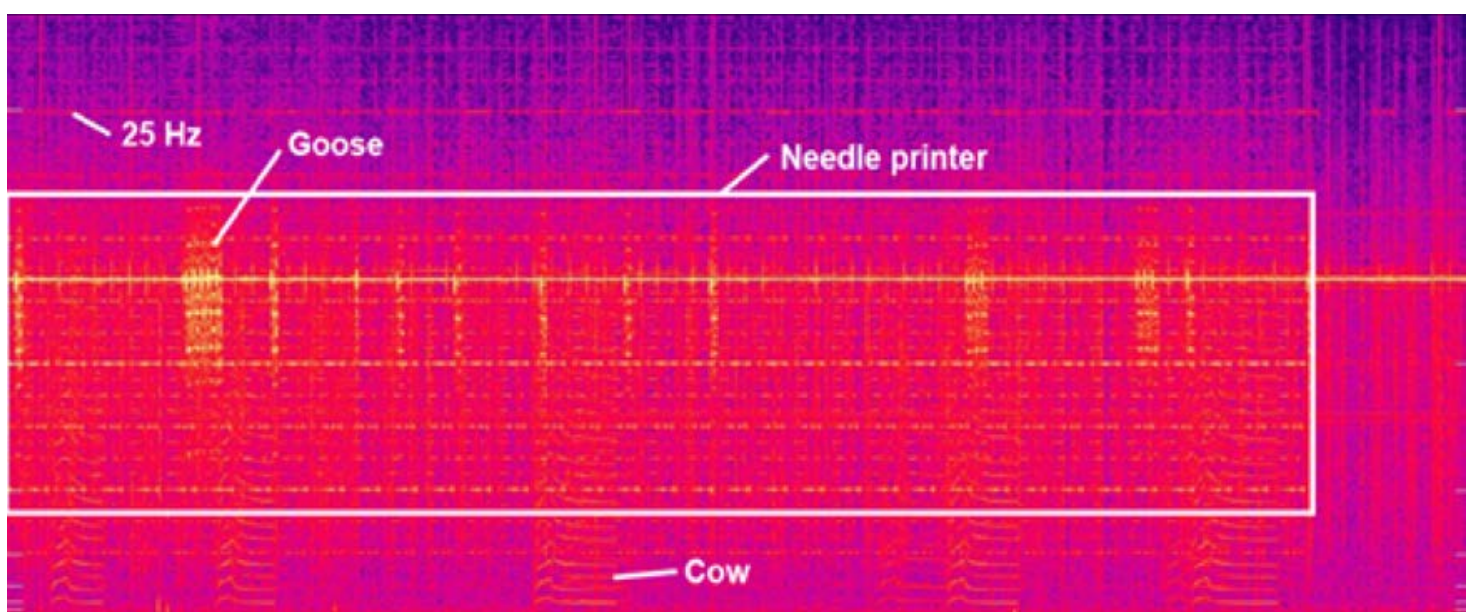

Fig. 8. The "needle printer" signal, frequency vs time; colour — as amplitude

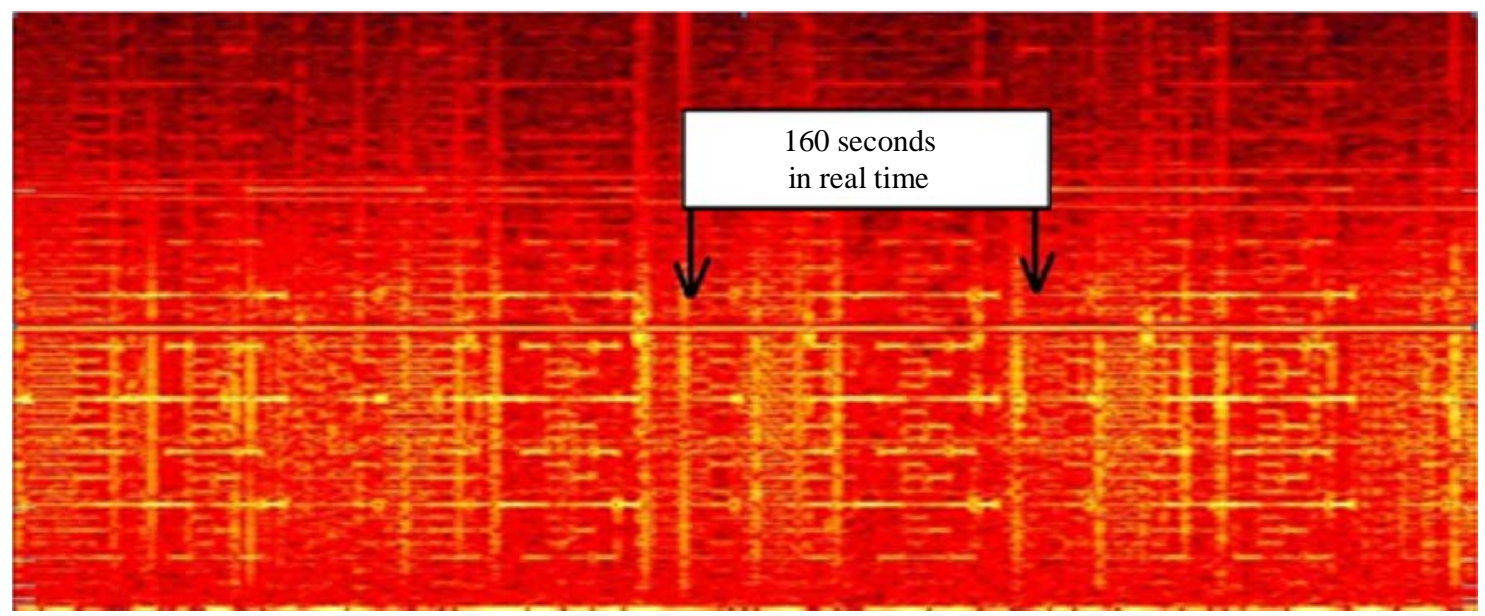

Fig. 9. One segment of a "dot-matrix" — signal frequency vs time; colour — as amplitude

at the coast of the North Sea, Hannover, Stuttgart, Bavaria, the Tankum lake, the Bigge lake and France (Mediterranean Coast).

Evaluation shows that in areas with dense population there are more kinds of signal and more intensive signal sources than in rural areas. There have been recordings inside large cities, suburbs, villages but even in the fields and in the woods and, in addition, at specific locations such as coastlines, lakes, on the edge of mining equipment, industrial plants and in front of household devices.

Because of the long wavelengths $(>6000 \mathrm{~km}$ ) and the used low-pass filter, the exact position of the receiver coil is not so crucial. It can be placed outdoors or inside in a big house, it is not important in general. However, the angle to the horizontal at which the coil is placed has a strong influence upon the measured amplitude of a certain signal. By adjusting the angle, locating can be made, eg to find the position of an ELF source. For that, a tiltand rotatable coil carrier was made.

A few signals can be detected over large areas (up to $30 \mathrm{~km}$ ). Most of them can be localized in a radius of just some hundred meters. In the case of domestic appliances or most industrial apparatus it is even shorter. Each place has its special signal mix. However, the types of most signals are similar. The occurrences in time vary between just ones for some hours up to 24 hour presence over years. Most signals occur daily for some hours. No correlation could be found between the signal content and temporal occurrence or spatial propagation. The recorded signals were given names based on the sound during audible playback.

The "goose" signal seems to contain some information. It has very likely an anthropogenic provenance as being measured in a large area (over $30 \mathrm{~km}$ ), 24 hours a day, with small gaps.

A similar temporally occurrence is typical for the "cow" signal. The spatial propagation is maybe not so distinctive. However, the time and frequency structure of this signal is completely different from the "goose" signal. A content which looks like included information cannot be seen. This signal can be of natural origin. It resembles "volcanic tremors", which is known by geologists as a seismic signal. It occurs in the neighbourhood of volcanoes.

Similarly, there are signals with just local spread which look very technical and others which look more natural. 


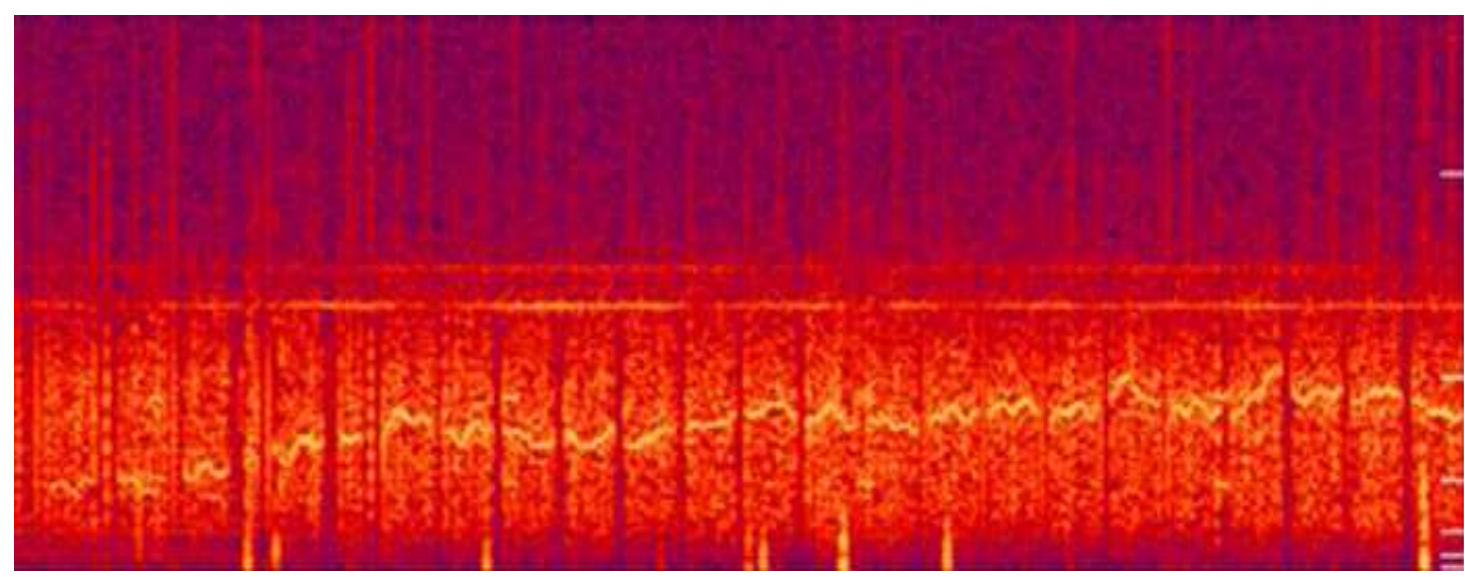

Fig. 10. The "whistle" signal: frequency vs time; colour — as amplitude

Table 1. Mainly used frequency and time resolution

\begin{tabular}{ccc}
\hline $\begin{array}{c}\text { FFT Window } \\
\text { size }\end{array}$ & $\begin{array}{c}\text { Frequency } \\
\text { Resolution } \\
\text { (samples) }\end{array}$ & $\begin{array}{c}\text { Time } \\
\text { resolution } \\
\Delta f(\mathrm{~Hz})\end{array}$ \\
\hline 1024 & 0.195 & 5.12 \\
2048 & 0.098 & 10.24 \\
\hline
\end{tabular}

A similar result can be found in the frequencies and the time structure. Very different structures are to be seen. Most signals include lot of frequencies. The typical structure is like one fundamental frequency with harmonics. Some signals contain only one frequency line (monophonic). However, in most signals the frequencies are not constant, they vary depending on time. Most signals consist of time patterns, typically repeating parts with periodic or non-periodic structures.

\section{EXAMPLES OF ELF-SIGNALS}

Signal alone cannot be analyzed in detail, however frequency transformation of time-window signals promise some results. We used the fast Fourier transformation with window size $w=1024$ and 2048 samples. The frequency (or the time) resolution $\Delta f$ (or $\Delta t$ ) for the original signal is then given by ratio (or its inverse) of sampling rate $f_{\mathrm{S}}$ to windows size $w$

$$
\Delta f=\frac{f_{\mathrm{S}}}{w}=\frac{1}{\Delta t}=\frac{f_{\mathrm{r}}}{s w} .
$$

As already mentioned the shifted sample rate (used by analyzing software) was $f_{\mathrm{T}}=32000 / s$, while $s=f_{\mathrm{T}} / f_{\mathrm{S}}=$ 160 , see Tab. 1, for details.

The following three examples are shown. The first one seems to have a natural origin. The second signal is assumingly caused by the humans, but maybe accidental and not planned. The third one, however, reminds communication. In fact, communication in this low frequency range is not efficient.

\section{The "cow" signal}

The "cow" signal (sound like a moo of a cow, hence the name) is one of the signals which may have a natural origin. It has medium propagation and was measured in the north of the Eifel Mountains. The Eifel is a low mountain. It lies between the cities Aachen in the north, Trier in the south and Koblenz in the east. The Eifel is a volcanic region. In the past, there were lots of volcanoes here. Actually there are above average seismic activities, like gas outlet at different places and movement of the ground.

The name "cow" results from the sound which appears if the signal is played through an audio-channel with 160 times speed. In Fig. 6 a constant repeating pattern of parallel lines can be seen between the lower edge of the diagram and the $16 \mathrm{~Hz}$ line and beyond. These are the "cow" signals. Each has a duration between 5 and 10 minutes. Figure 7 shows a single cow signal with duration of 8 minutes. The signal consists of a varying fundamental frequency of $0.8 \mathrm{~Hz}$ and more than 20 harmonics. The vertical distance between the lines is constant. It is typical for this signal that the frequency at the beginning of the signal increases gradually, then gradually decreases and then remains at a constant frequency until it ends abruptly.

Comparing the signal with the seismic signal "volcanic tremors", a striking resemblance can be seen. So it is obvious that the "cow" signal comes from seismic activities.

\section{The "needle printer" signal}

This signal appeared late December 2004, for the first time at the measurement location Hrtgenwald and could be received in May 2005 for several days almost constantly, see Fig. 8. In Fig. 9 a repeating structure can be seen. The basic element has a duration of 160 seconds in real time. In the picture four basic elements can be seen. The listened sound of a basic element reminds of the sound of an old fashioned dot matrix printer.

A strong zoomed display of seven individual elements in the time domain shows that this signal includes a very 


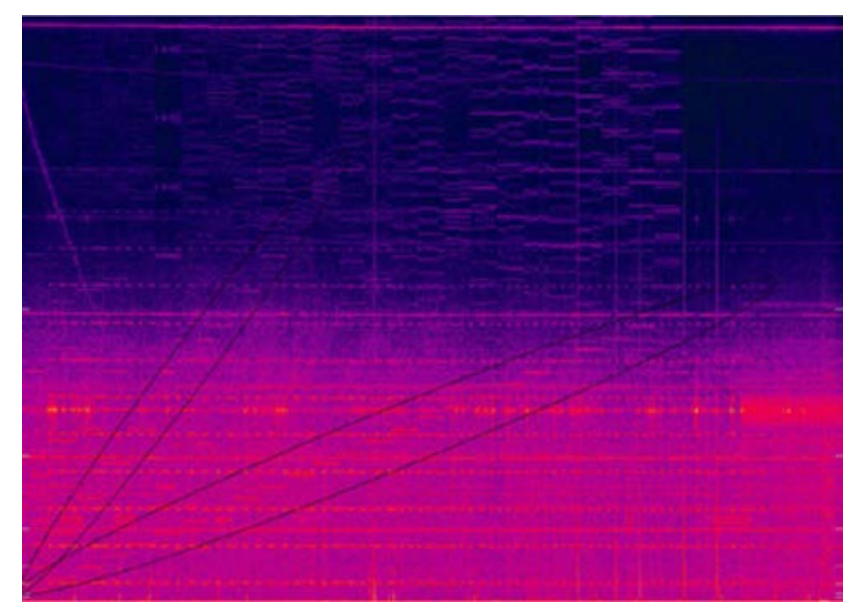

Fig. 11. "Natural" ELF-Signals and artificial ELF-Signals (marked) in one diagram

long sequence of pulses with different spacing from each other. The signal begins with a pulse sequence which has a distance of about 2.6 seconds. The intervals between the pulses become shorter and shorter until they have a length of only $160 \mathrm{~ms}$. Then it proceeds in reverse order: The pulses are getting longer and then again getting shorter. The structure of the "needle printer " signal suggests an anthropogenic origin.

\section{The "whistle" signal}

The frequency range of this signal is always below $16 \mathrm{~Hz}$. When listening to it $(16 \mathrm{~Hz} \times 160=2560 \mathrm{~Hz})$, the signal sounds like a flute player who plays a melody. The length of each pattern and sequences between tones and pauses is constant. But the tones sequences are not consistent. Sometimes, groups with 11 to 17 tones can be detected with a short interruption, sometimes without interruption, Fig. 10. The sounds follow a line that moves up and down. It is striking that the sounds during the short duration of a whistle change slightly in their frequency: it rises, falls or returns to the original pitch. The following whistle starts exactly with the frequency with which the previous one ends. Is this a rising or a falling tone, the result is a rising or falling frequency line.

Between longer signal pauses, the same signal structure can be received again but with lower signal strength - as if a second station replied. Maybe the information could be in the design of the individual frequency bows. Different patterns are clearly distinguishable.

The whistle signal allows the hypothesis of cypher transmission. All signals from the year 2004 were analyzed on their typical patterns. Note that the patterns are made by frequency change (frequency modulation). The characters are into their distribution pretty evenly. The most occurring patterns are 1 " wavy line" with a positive transition

2 " wavy line" with a negative transition

3 "/" slash

4 " " slash reversed

5 "v" letter
6 "^", circumflex

7 "_" line

Other characters are less striking.

\section{SIMULATION}

To verify the measurements, the following simulation was conducted

(a) Artificial ELF signals were emitted with the help of a wired loop.

(b) Currents with different frequencies were feed in the ground of a garden with the help of rods.

The described acquisition devices were used to record the resultant ELF waves. The signal created by a voltage generator was sent into an amplifier which delivering peak currents up to $8 \mathrm{~A}$ at output voltages up to $\pm 38 \mathrm{~V}$. The waveform of this signal was observed with an oscilloscope.

Figure 11 shows one example of the simulation. The amplitude of the artificial signal was set in a way that it has the same colour in the diagram like the "natural" signals. Furthermore, the frequency was increased stepwise. The result is a frequency stair in the diagram. It is noteworthy that the artificially produced signal has a similar structure like the "natural" signals. With the help of this simulation it could be determined that the magnetic induction of the original signals was in the range of $1 \mu \mathrm{T}$ at the coil. Also, it could be determined that most of the "natural" signals have a non-sinusoidal structure.

\section{THE EFFECT OF ELF-SIGNALS}

Strange behaviour of analogue circuits can be caused by ELF signals.

For instance, signal disturbances at CERN, CMS-VDC experiment in the service cavern UXC in the years 2005 to 2010 were caused by ELF signals. The effect was slow disturbing signals, superimposed on the signals of analogue circuits, as well as effects like "floating ground".

Rattle noise came out from an audio amplifier. With a parallel made measurement it could be shown that this rattle noise was caused by an ELF signal.

In an exchange of views with the geophysicist [13] it came to light that it is very likely that geologists have accidentally recorded ELF signals during geodesy. This was then classified as "technical trouble" and not further pursued.

Other influences from ELF signals, maybe on living organism (fauna and flora) can be examined now with regard to the structure of the ELF signals.

\section{IDEAS ABOUT THE SOURCES OF THE SIGNALS}

After publications $(e g[9,10])$ lots of suggestions about the signal sources came from the readers. Almost all proposals were tracked but did not lead to concrete result. 
For example one reader wrote that some of the signals were from submarine communication. Careful researches could not confirm this. Rather, the submarine communication works on frequencies higher than $50 \mathrm{~Hz}$. Another example was a reader who said that this signals originated from lignite mining between Aachen and Cologne. So, the author made measurements in front of a mining area. Speculations of the reader could not be confirmed.

So, the authors developed his own ideas. One idea is that electrical leakage currents of the power supplies and also natural sources from the ground are collected by pipelines. Because of the collection, the current in the pipelines is so large that the emitted fields could be measured over long distances. Some of the signals could be explained in this way,- but not all. A further question is: if this idea works, where do the very low frequencies come from? However, this work is not to find the sources, rather to show, that ELF signals exist and how they look like.

The currents in DC engines of trams change depending on the speed. Trams with AC motors are supplied through AC/AC-converters. Stray currents from these motors could be accumulated by pipelines. Stray currents are very likely in this case because the return-current flows through the rails.

Outside of areas inhabited, only a few signals can be measured. One of a "natural candidate" is the cow signal described above. One idea of a natural ELF generator in the ground is the OGS (oscillating geological structure). A piezoelectric sensitive structure in the ground is cyclically pressed and relaxed through seismic energy. In this way it produces electric current. The current flows through the conducting soil layers or produces stray currents which will be collected by underground cables, electrically conductive pipelines or creeks/rivers, which leads to the emission of ELF magnetic fields.

\section{REFERENCES}

[1] FRASER-SMITH, A. C.: Reception of ELF Signals at Antipodal Distances, Radio Science 33 No. 1 (1998), 83-88.

[2] BARR, R.-LLANWYN, J. D.-RODGER, C. J.: ELF and VLF Radio Waves., Journal of Atmospheric and Solar-Terrestrial Physics 62 (2000), 1689-1718.

[3] LIPKOVA, J.-CECHAK, J.: Human Electromagnetic Emission in the ELF Band, Measurement Science Review 5 Sec. 2 (2005), 29-32.

[4] MASAKI, Y.-KOZO, Y.-YUICHI, I.-HAYAKAWA, M.NICKOLAENKO, A. P.: Ionospheric Monitoring by ELF Signals Received at Moshiri Station in Japan, In: SICE Annual Conference, The University Electro-Communications (Japan), 2008, pp. 1678-1682, PR0001/08/0000-1678 Y400 (c) 2008 SICE.

[5] SHAO-MING, H.-WANG, K.-CHEN, A. B. C., HANTZONG, S.-SUNG-MING, H.-RUEI-RON, H-TUNGSHIN, H.: The Characteristics of Magnetic Signals Observed at Lulin ELF Station and their Association with Earthquakes in Taiwan, Terr. Atmos. Ocean. Sci. 24 No. 2 (2013), 197-206, doi: 10.3319/TAO.2012.09.19.01(SEC).
[6] ZWICKER, E.-FELDKELLER, R. : Das Ohr als Nachrichtenempfänger (The ear as a message recipient), ASIN: B0000BUCGM, Publishing House Hirzel, 1967. (in German)

[7] ZANTIS, F. P.: "Psychoacoustics", Cyclical lesson at "HEAD acoustics GmbH" TrainingCenter, Herzogenrath (Germany) in 2000-2011.

[8] ZANTIS, F. P.: Schwache, niederfrequente magnetische Wechselfelder mit linearisierten Spulen erfassen (Weak, Low-Frequency Magnetic Alternating Fields, Detecting with Linearized Coils), Magazin 'WissenHeute' (May 2009), 31-38. (in German)

[9] DIEDRICH, K.-ZANTIS, F. P.: Erforschung schwacher magnetischer Wechselfelder, Teil 1 (Researching of Weak Alternating Magnetic Fields, Part 1), CQDL (May 2006), 334-337. (in German)

10] DIEDRICH, K.-ZZANTIS, F. P.: Erforschung schwacher magnetischer Wechselfelder, Teil 2 (Researching of Weak Alternating Magnetic Fields, Part 2), CQDL (June 2006), 419-422. (in German)

[11] ZANTIS, F. P.: Erfassung und Auswertung von schwachen, niederfrequenten, magnetischen Wechselfeldern in unserer Umwelt (Collection and Analysis of Weak, Low-Frequency Alternating Magnetic Fields in Our Environment), GRIN Publishing House, 2010. (in German)

12] BELICH, U.: Grundlagen der elektrochemischen Korrosion und des Korrosionsschutzes (Basics of Electrochemical Corrosion and the Corrosion Protection), Unterrichtsblätter der Deutschen Bundespost 31 No. 9 (10 Sep 1978). (in German)

[13] Private communication with Prof. Erhard Wielandt (University of Stuttgart).

Received 7 January 2016

Franz Peter Zantis was born in 1959. He received his Dipl-Ing from the Aachen University of Applied Sciences in 1985. He worked as engineer in the industry with an emphasis on the field of sound and vibration analysis (NVH). Since 2011 he has been at the RWTH Aachen University. In 2012 he received the degree Dipl-Ing from the Slovak University of Technology. He started researching of ELF signals in 2000.

Ján Hribik (Assoc Prof, Ing, PhD) was born in 1948. He graduated from the Slovak Technical University, Faculty of Electrical Engineering in Bratislava in 1971. Since 1972 he has been with the Faculty of Electrical Engineering and Information Technology, Slovak University of Technology in Bratislava. Here he also received the $\mathrm{PhD}$ degree in Electronic Engineering in 1981. In 1989 he was appointed Associated Professor. His professional activities concern electronic measurements and analogue electronic circuits, namely in the field of integrating AD conversion especially current-to-frequency conversion. Since 1996 his activities concern mainly electric power and energy measurement.

Daniela Ďuračková (Prof, Ing, PhD) was born in 1950. She received her Ing from the Slovak University of Technology in 1974, where she received also PhD degree in 1980. Since 1979 she has been with the Department of Microelectronics, now the Institute of Electronics and Photonics, since 2004 as professor. Her research interests changed from solid state physics towards VLSI analogue and digital design on a chip. In 2004 she was awarded as the "Technologist of the Year" by the Ministry of Education of Slovak Republic for the pioneer design of two neuro-chips in Slovakia. Her professional activities concern mainly design of electronic VLSI circuits. 